\title{
Neuroprotective Effects of Pycnogenol Against Oxygen-Glucose Deprivation/ Reoxygenation-Induced Injury in Primary Rat Astrocytes via NF-KB and ERK1/2 MAPK Pathways
}

\author{
Ruixue Xia Chunxue Ji Leguo Zhang \\ Department of Neurology, Cangzhou Center Hospital, Cangzhou, Hebei, China
}

\section{Key Words}

Pycnogenol • Oxygen-glucose deprivation/reoxygenation • Oxidative stress • Inflammatory response - Astrocytes

\begin{abstract}
Backgrounds/Aims: Pycnogenol (PYC) is a patented mix of bioflavonoids with potent antioxidant and anti-inflammatory properties. In this study, we investigated the effects of PYC on oxygen-glucose deprivation/reoxygenation (OGD/R)-induced injury in primary rat astrocytes. Methods: The primary rat astrocytes were randomly divided into 6 groups, blank control, OGD/R, OGD/R+PYC $(10,20,40$, and $60 \mu \mathrm{g} / \mathrm{mL})$. The cell activity were detected by MTT and LDH assays, then the levels of oxidant products [malondialdehyde (MDA) and reactive oxygen species (ROS)], antioxidants [superoxide dismutase (SOD)], mitochondrial membrane potential (MMP) and inflammatory cytokines were detected. In addition, the expression levels of apoptosis-related proteins (Bax, Bcl-2 and Cleaved caspase 3), proinflammatory factors (NF-KB p65), and p-ERK1/2 were measured by Western blot analysis. Results: The results showed that PYC incubation dose-dependently attenuated cell viability loss, LDH leakage, oxidative stress, inflammatory cytokines accumulation and cell apoptosis caused by OGD/R. Furthermore, PYC pretreatment dose-dependently suppressed OGD/R-induced NF-KB p65 nuclear translocation, NF-KB activity and ERK1/2 phosphorylation. Similarly to PYC, NF-KB inhibitor PDTC and ERK1/2 inhibitor PD098059 dramatically inhibited OGD/R-induced NFKB activation, ERK1/2 phosphorylation, and ROS production, as well as TNF- $\alpha$ secretion. Conclusions: These findings revealed that PYC has neuroprotective effects against OGD/Rinduced injury via NF-KB and ERK1/2 pathways in primary rat astrocytes.
\end{abstract}




\section{Cellular Physiology Cell Physiol Biochem 2017;42:987-998 \begin{tabular}{l|l} 
and Biochemistry $\begin{array}{l}\text { DOI:1159/000478681 } \\
\text { Published online: June 27, } 2017\end{array}$ & $\begin{array}{l}\text { (c) } 2017 \text { The Author(s). Published by S. Karger AG, Basel } \\
\text { www.karger.com/cpb }\end{array}$
\end{tabular}}

Xia et al.: Protective Effects of Pycnogenol in Rat Astrocytes

\section{Introduction}

Ischemic stroke is one of the leading causes of adult death and disability worldwide. Astrocytes are the most abundant glial cells in the central nervous system and provide structural and nutritive support for neurons [1]. In ischemic stroke, astrocytes are involved in the regulation of water and ion homeostasis, cerebral blood flow, maintenance of the blood brain barrier, and control of the extracellular level of glutamate, as well as being a source of neuroprotectants. On the other hand, astrocytes may also enlarge the ischemic area due to their participation in inflammatory processes and production of potential neurotoxic substances [2]. Additionally, impairment or loss of astrocytes may result in neuronal death or dysfunction [3]. Astrocytes contribute to proper neuronal function during both physiological and pathological conditions. Therefore, protection against astrocytes injury has become an important strategy in the development of novel neuroprotective therapies for ischemic stroke.

Pathologically, ischemic stroke is characterized by initial excitotoxicity and ensuing secondary complications such as oxidative damage, inflammation, and apoptosis. Astrocytes comprise the structural architecture of the brain and support neurons to maintain homeostasis [3]. As brain tissue is more likely to produce free radicals and lipid peroxides, oxidative stress is likely to be the key factor responsible for the irreversible damage caused during cerebral ischemia/reperfusion. Previous studies suggest that oxidative stress is a fundamental mechanism of cerebral ischemia/reperfusion injury and occurs due to an imbalance between reactive oxygen species (ROS) production and the cell's capacity to neutralize this via its intrinsic anti-oxidant defense system. It has been confirmed that oxidative stress can affect the glial cells (astrocytes and microglia), which play a complex role in dopaminergic neurotoxicity [4]. Both microglia and astrocytes can degrade dopamine with the subsequent generation of oxidative stress and detrimental effects on dopaminergic neurons [5]. In addition, astrocytes appear to be important regulators of the inflammatory events that occur in stroke [6]. Accumulating evidence has demonstrated that inflammatory mediators released from necrotic neurons after stroke induce astrocyte activation, which in turn produces cytotoxic substances and induces astrocytic inflammatory response to aggravate the ischemic damage [7]. It has also been demonstrated that astrocytes in primary culture undergo apoptosis following an ischemic insult [8]. Thus, agents with anti-oxidant, anti-inflammatory and anti-apoptotic properties may provide an effective therapeutic approach against astrocytes injury during ischemic stroke.

Pycnogenol (PYC) is a natural plant extract obtained from the bark of French maritime pine (Pinus pinaster). The main constituents of PYC are phenolic compounds, broadly divided into monomers and condensed flavonoids [9]. It has been reported that PYC possesses various biological activities, including anti-oxidant [10], anti-inflammatory [11], anti-cancer [9] and anti-platelet [12] activities, implying that PYC may have the potential to be used in the treatment of ischemic stroke. Previous study has showed the neuroprotective effect of PYC following traumatic brain injury in rats models [13] Moreover, PYC had been reported to protect human neuroblastoma SH-SY5Y cells following acrolein-induced cytotoxicity [14]. Hence, in the present study, we investigated the effects of PYC on oxygen-glucose deprivation/reoxygenation (OGD/R)-induced injury and further explored the underlying related molecular mechanism in primary rat astrocytes, an in vitro model of ischemic stroke.

\section{Materials and Methods}

Preparation of rat cerebral astrocytic cultures

The procedure used to dissociate rat astrocytes was similar to that described previously by Schaefer et al. [15]. Sprague-Dawley (SD) rats aged less than $48 \mathrm{~h}$ were supplied by the Experimental Animal Center of Hebei Medical University. All animal experimental procedures were performed in accordance with the Guide for the Care and Use of Laboratory Animals and were approved by the Experimental Animal Ethics 


\section{Cellular Physiology Cell Physiol Biochem 2017;42:987-998 \begin{tabular}{l|l|} 
and Biochemistry Published online: June 27, 2017 & $\begin{array}{l}\text { C } 2017 \text { The Author(s). Published by S. Karger AG, Basel } \\
\text { www.karger.com/cpb }\end{array}$ \\
\hline
\end{tabular}}

Xia et al.: Protective Effects of Pycnogenol in Rat Astrocytes

Committee of Hebei Medical University, China. Briefly, meninges-free cortices were cut into small cubes and digested with trypsin at $37^{\circ} \mathrm{C}$. After being mechanically dissociated, the cell suspension was sieved through nylon filters. Then the cultures were maintained in DMEM/F12 (Gibco, Rockville, MD, USA) with 10\% fetal bovine serum (FBS; Gibco), $100 \mathrm{U} / \mathrm{mL}$ penicillin, and 100 mg/mL streptomycin (Life Technologies, Rockville, MD, USA) in an incubator with a humidified atmosphere of $5 \% \mathrm{CO}_{2}$ and $95 \%$ air at $37^{\circ} \mathrm{C}$ for $2-3$ weeks, during which the medium was changed twice per week. The cells were labeled with immunofluorescent glial fibrillary acidic protein (GFAP; Wuhan Boster Biological Technology Co. Ltd., Wuhan, China), which is specific for astrocytes, to evaluate culture purity. Cultures with a purity of at least $95 \%$ were used in the study.

Flow cytometry

For intracellular GFAP and CD11b staining, rat primary astrocytes cultures were washed and detached in a non-enzymatic manner, spun and resuspended in a cell permeabilization/fixation buffer for $30 \mathrm{~min}$ on ice, washed in PBS and then blocked in a permeabilization buffer (R\&D biosciences) containing 1\% FCS and 10\% Fc Blocker solution (Miltenyi Biotech) for $10 \mathrm{~min}$. Anti-GFAP-FITC (1:40) and anti-CD11b-PE antibody (1:100, Abcam, Cambridge, UK) was added to the blocking solution for 30 min. Finally, the cells were resuspended for flow cytometric analysis [16].

\section{OGD/R procedure and drug treatment}

We established an in vitro model of cerebral ischemia by subjecting cells to OGD. Briefly, cultured astrocytes were washed twice with phosphate buffered saline (PBS) and incubated in DMEM/F12 without glucose. Astrocytes were then placed into a specialized, humidified chamber filled with $92 \% \mathrm{~N}_{2} / 5 \% \mathrm{CO}_{2} / 3 \%$ $\mathrm{O}_{2}$ at $37^{\circ} \mathrm{C}$ for $6 \mathrm{~h}$ in order to establish conditions of OGD. Thereafter, the culture medium was replaced with normal DMEM/F12 containing glucose under normoxic conditions for an additional $24 \mathrm{~h}$ for OGD/R.

PYC powder was obtained from Henkel Corporation (La Grange, IL, USA) and dissolved in DMSO. PYC $(10,20,40,60 \mu \mathrm{g} / \mathrm{mL})$ was applied to the cell cultures $24 \mathrm{~h}$ prior to OGD/R exposure. Either $25 \mu \mathrm{M}$ nuclear factor-kappaB (NF- $\kappa$ B) inhibitor pyrrolidine dithiocarbamate (PDTC) or extracellular signal-regulated kinase 1/2 (ERK1/2) inhibitor PD098059 (Sigma, St. Louis, MO, USA) was introduced to the cell cultures 30 min before PYC pretreatment. The cultures in the blank control group were maintained in normal DMEM/ F12 medium under normoxic conditions at $37^{\circ} \mathrm{C}$ without OGD/R exposure and PYC pretreatment. The cultures in the control group were subjected to OGD/R in the absence of PYC pretreatment.

\section{MTT assay}

Cell viability was measured using the MTT assay. Briefly, the cultured cells $\left(5 \times 10^{3}\right)$ were seeded into 96-well plates (Corning Inc., Corning, NY, USA) in DMEM/F12 with $10 \% \mathrm{FBS}$ for $24 \mathrm{~h}$ at $37^{\circ} \mathrm{C}$ in $5 \% \mathrm{CO}_{2}$. After exposure to OGD/R, $20 \mu \mathrm{L}$ modified tetrazolium salt MTT ( $5 \mathrm{mg} / \mathrm{mL}$; Sigma) was added to each well and the samples were incubated at $37^{\circ} \mathrm{C}$ for $4 \mathrm{~h}$. The supernatant was then carefully removed and $100 \mu \mathrm{L}$ DMSO was added to lyse the cells. Once the dark-blue MTT crystals had dissolved, the absorbance was measured at 490 nm using a Benchmark microplate reader (Bio-Rad, Hercules, CA, USA).

\section{Lactate dehydrogenase ( $\mathrm{LDH}$ ) release assay}

Astrocytes injury was quantitatively assessed by measuring LDH activity released from damaged or dead cells using the LDH activity assay kit following the manufacturer's instructions. Previous findings have shown that the activity of LDH from either necrotic or apoptotic cells is proportional to the number of neurons damaged or destroyed. Briefly, the cells $\left(5 \times 10^{3}\right)$ were seeded in 96-well plates (Corning Inc.) and incubated for $24 \mathrm{~h}$. After OGD/R exposure, $50 \mu \mathrm{L}$ medium was removed and the amount of LDH leakage from the cells was determined at $490 \mathrm{~nm}$ using a microplate reader (Bio-Rad). The results were expressed as the percentage of LDH release relative to the control cells.

Measurement of cellular superoxide dismutase (SOD) and malondialdehyde (MDA) levels

After OGD/R exposure, cultured cells were harvested, washed twice, sonicated on ice, centrifuged, and then the supernatants were collected. Cellular SOD levels were assayed by a modification of the xanthine/ xanthine oxidase method [17] and determined by spectrophotometry using a commercial kit (Jiangcheng Bioengineering, Nanjing, China). The levels of MDA, a compound produced during lipid peroxidation, were 


\section{Cellular Physiology Cell Physiol Biochem 2017;42:987-998 \begin{tabular}{l|l} 
DOI: 10.1159/000478681 & a 2017 The Author(s). Published by S. Karger AG, Basel \\
www.karger.com/cpb
\end{tabular} \\ Xia et al.: Protective Effects of Pycnogenol in Rat Astrocytes}

measured based on a reaction with thiobarbituric acid using a commercial kit (Beyotime, Shanghai, China) [18]. The optical density at $532 \mathrm{~nm}$ was determined using a microplate reader (Bio-Rad).

\section{Measurement of ROS content}

ROS formation was determined with the fluorescent probe 2',7'-dichlorfluorescein-diacetate (DCFHDA) using a commercial kit (Jiangcheng Bioengineering). Cell-permeable non-fluorescent DCFH-DA is oxidized to the highly fluorescent 2,7-dichlorofluorescein in the presence of ROS. The cells were washed twice with PBS, and then incubated with $10 \mathrm{mM}$ DCFH-DA for $30 \mathrm{~min}$ at $37^{\circ} \mathrm{C}$ in the dark. The cells were then harvested and suspended in PBS. The fluorescence intensity was measured using a fluorospectrophotometer (Hitachi, Tokyo, Japan) at excitation/emission maxima of 485/525 nm [19].

\section{Mitochondrial membrane potential (MMP or $\triangle \psi m$ ) determination}

The cationic dye JC-1 was used to evaluate MMP loss in OGD/R-treated astrocytes using a commercial kit (Beyotime) under a fluorescence microscope (Olympus, Tokyo, Japan). JC-1 is a convenient voltage sensitive probe used to monitor MMP [20]. The cells containing J-aggregates have high $\Delta \psi \mathrm{m}$ and show fluorescence. JC-1 maintains its monomeric form in those cells with low $\Delta \psi \mathrm{m}$, which show only green fluorescence [21]. The relative proportions of red and green fluorescence were used to measure the ratio of mitochondrial depolarization.

\section{Enzyme-linked immunosorbent assay (ELISA)}

After OGD/R exposure, the release of pro-inflammatory cytokines (TNF- $\alpha$, IL-1 $\beta$, and IL-6) from cellular supernatant was analyzed in triplicate using specific ELISA kits (R\&D Systems, Minneapolis, MN, USA) according to the manufacturer's instructions. The absorbance at $450 \mathrm{~nm}$ was determined using a microplate reader (Bio-Rad).

\section{Cell apoptosis assay}

Cell apoptosis was measured by flow cytometry analysis as described previously [22]. Treated cells were harvested and re-suspended in $2.5 \mathrm{ml}$ phosphate buffered solution (PBS). After incubation with a mixture containing Annexin V-FITC and PI (Roche Diagnostics, USA) in binding buffer for $15 \mathrm{~min}$ in the dark, the stained cells were counted using flow cytometry (BD, Mountain View, USA). Annexin V-FITC binds to cells that express phosphatidylserine on the outer layer of the cell membrane, and PI stains the cellular DNA of cells with a compromised cell membrane.

\section{$N F-\kappa B$ assay}

After OGD/R exposure, the cells were washed with cold PBS, collected by centrifugation, resuspended in hypotonic lysis buffer, incubated on ice for $15 \mathrm{~min}$ and then mixed with $0.75 \%$ Nonidet P-40 (NP-40) solution. The nuclear extract was collected and centrifuged at 4,000 $\times$ g for $2 \mathrm{~min}$, and then stored at $-80^{\circ} \mathrm{C}$. Then, NF- $\mathrm{kB}$ activity in nuclear extracts was analyzed using an NF- $\mathrm{\kappa B}$ p65 ActivELISA kit (Imgenex, San Diego, CA, USA) according to the manufacturer's instructions. The absorbance was determined at $405 \mathrm{~nm}$ using a microplate reader (Bio-Rad).

\section{Preparation of nuclear and cytoplasmic extractions}

Nuclear and cytoplasmic extractions were performed using the Nuclear and Cytoplasmic Extraction kit (Beyotime) following manufacturer's instructions [23]. The cells were harvested and washed with $1 \mathrm{ml}$ buffer A ( $1.5 \mathrm{mM} \mathrm{MgCl}_{2}, 10 \mathrm{mM}$ HEPES, $18 \mathrm{mM} \mathrm{KCl}$ ) for $5 \mathrm{~min}$ at $600 \mathrm{~g}$. And then resuspended in buffer A and $0.1 \% \mathrm{NP} 40$, left for $10 \mathrm{~min}$ on ice to lyse the cells and centrifuged for $3 \mathrm{~min}$ at $600 \mathrm{~g}$. The supernatant was saved as cytosolic extract. Washed the nuclear pellet in $1 \mathrm{ml}$ buffer A for $3 \mathrm{~min}$ at $4000 \mathrm{~g}$, resuspended in 30 $\mu l$ buffer C (25\% glycerol, $0.42 \mathrm{M} \mathrm{NaCl}, 20 \mathrm{mM}$ HEPES pH 7.9, $1.5 \mathrm{mM} \mathrm{MgCl}{ }_{2}, 0.2 \mathrm{mM}$ EDTA), rotated at $4{ }^{\circ} \mathrm{C}$, then centrifuged for $20 \mathrm{~min}$ at $14,000 \mathrm{~g}$. The supernatant was saved as nucleus extract.

\section{Western blot analysis}

Cell lysates were collected and protein concentrations were determined using the Pierce BCA Protein Assay Kit (Thermo Scientific, Rockford, IL, USA). Equal amounts of protein were processed for western blotting according to the standard protocols. The primary antibodies used were anti-cleaved caspase- 3 
antibody (Cell Signaling Technology, Beverly, MA, USA); anti-Bax, anti-Bcl-2, anti-p-p65, anti-p65, anti-pERK1/2, anti-ERK1/2, and anti- $\beta$-actin antibodies (Santa Cruz Biotechnology, Santa Cruz, CA, USA). The protein bands resulting from incubation with proper secondary antibody were visualized by ECL (Beyotime). The absorbance values of target proteins were determined using Gel-Pro Analyzer version 4.0 software (Media Cybernetics, Silver Spring, MD, USA).

\section{Statistical analysis}

Data were expressed as mean \pm SD of results derived from three independent experiments. All analyses were conducted using SPSS software (SPSS Inc., Chicago, IL, USA). Multiple comparisons were performed with one-way ANOVA followed by LSD test. $P<0.05$ was considered statistically significant compared to the respective control.

\section{Results}

PYC pretreatment prevented OGD/R-induced loss of cell viability in primary rat astrocytes Cell culture purity was assessed using flow cytometry. Flow cytometry quantification of GFAP protein, a known astrocyte marker, showed that $>95 \%$ of the population was GFAP positive. Moreover, we examined the presence of microglia in astrocyte cell culture by antiCD11b staining, and the results showed that there was an average of 5.6\% CD11b-positive cells in our astrocyte cell culture (Fig. 1A). In order to examine the protective effects of PYC against cell cytotoxicity caused by OGD/R, MTT and LDH assays were performed to detect the loss of cell viability in primary rat astrocytes. Compared with the blank control group, cell viability was significantly decreased after subjecting the cells to OGD/R, but this was restored by PYC $(10,20,40$, and $60 \mu \mathrm{g} / \mathrm{mL})$ pretreatment in a dose-dependent manner (Fig. 1B). Additionally, LDH leakage was increased after OGD/R exposure, and incubation with PYC attenuated OGD/R-induced LDH leakage in a dose-dependent manner (Fig. 1C). These results demonstrated that PYC pretreatment attenuated OGD/R-induced loss of cell viability in primary rat astrocytes.

PYC pretreatment suppressed OGD/R-induced oxidative stress in primary rat astrocytes

We then investigated the effects of PYC on OGD/R-induced oxidative stress in primary rat astrocytes. The results showed that the SOD activity was reduced after OGD/R exposure,

Fig. 1. Effects of PYC on cell viability in primary rat astrocytes subjected to OGD for $6 \mathrm{~h}$ followed by reoxygenation for 24 h. (A) Quantification of percentage of GFAP and CD11b positive cells in primary rat astrocytes culture by flow cytometry. (B) Cell viability was detected using the MTT assay. (C) LDH leakage was measured using a commercial LDH activity assay kit. ${ }^{*} P<0.05$ vs. blank control group, ${ }^{\#} P<0.05$ vs. control group.

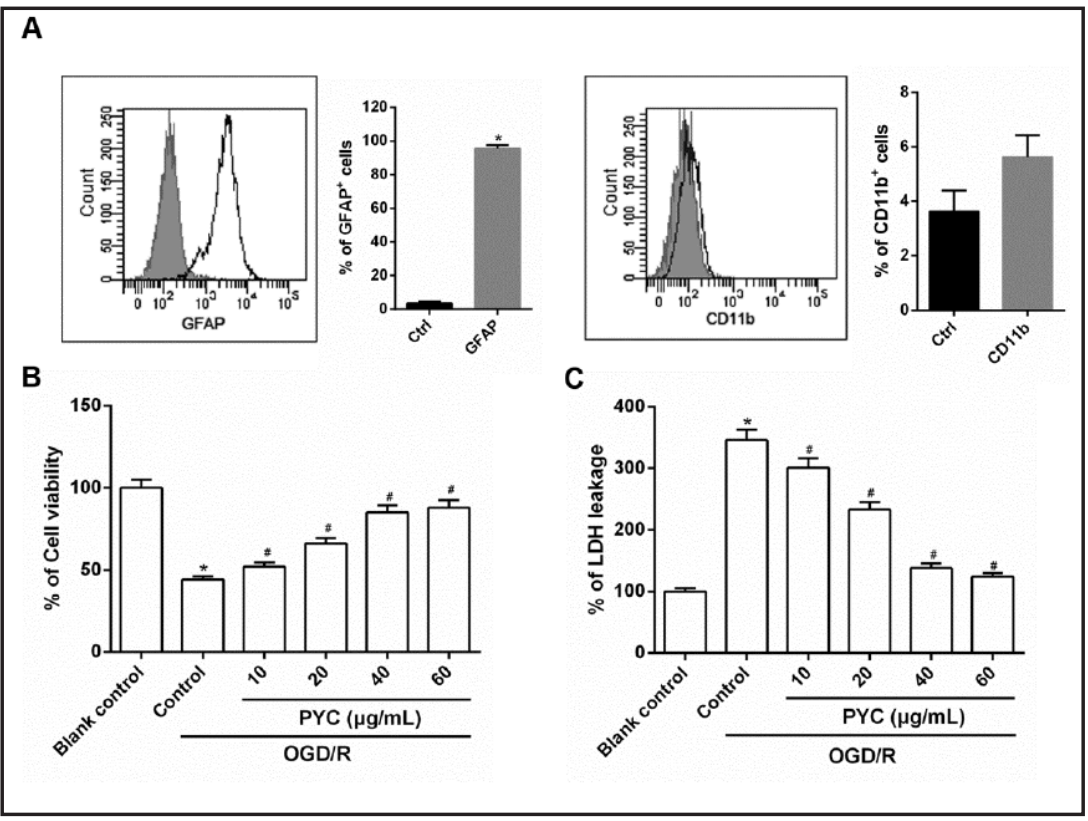


Fig. 2. Effects of PYC on the oxidative status in primary rat astrocytes subjected to OGD for $6 \mathrm{~h}$ followed by reoxygenation for 24 h. The levels of oxidative status markers including (A) ROS, (B) SOD, (C) MDA, and (D) MMP were determined using the commercial kits. ${ }^{*} P<0.05$ vs. blank control group, ${ }^{\#} P<0.05$ vs. control group.

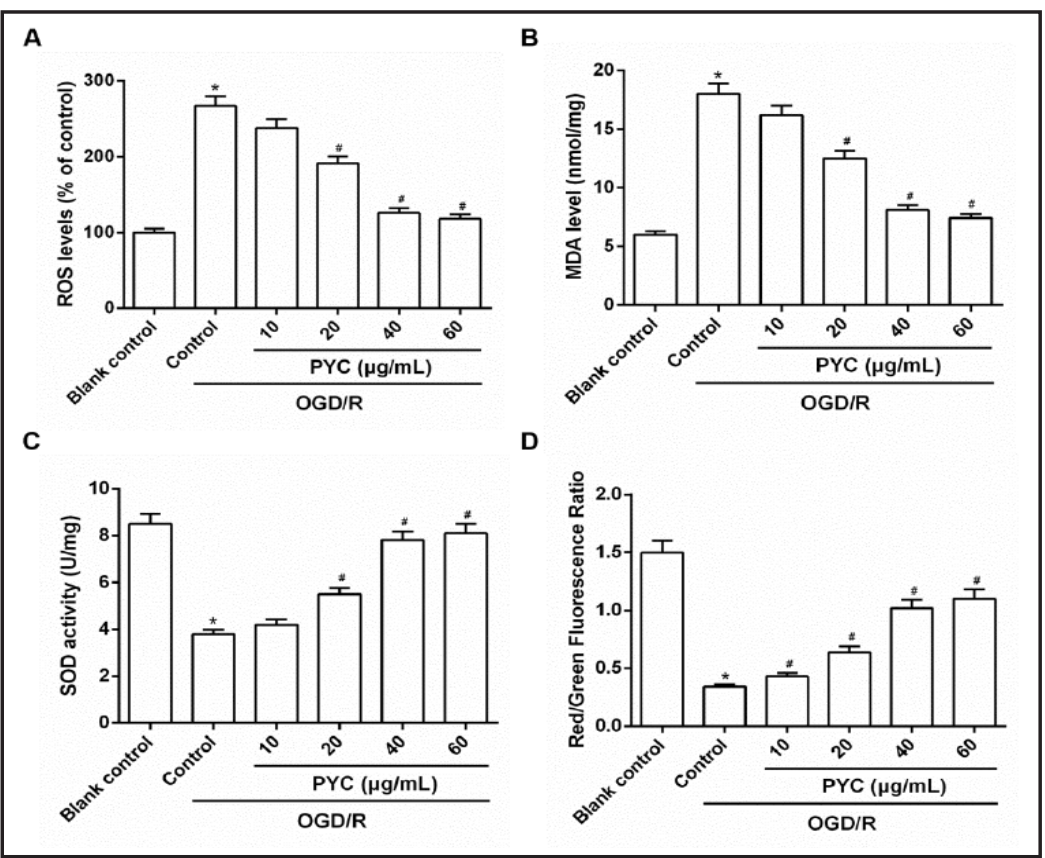

Fig. 3. Effects of PYC on the inflammatory response in primary rat astrocytes subjected to OGD for $6 \mathrm{~h}$ followed by reoxygenation for $24 \mathrm{~h}$. The levels of (A) TNF- $\alpha$, (B) IL-6, and (C) IL-1 $\beta$ were determined using the commercial kits. ${ }^{*} P<0.05$ vs. blank control group, ${ }^{\#} P<0.05$ vs. control group.
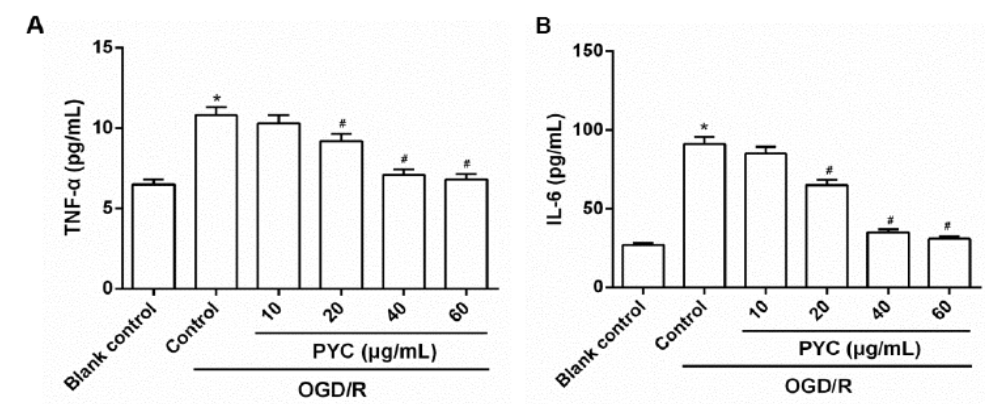

C

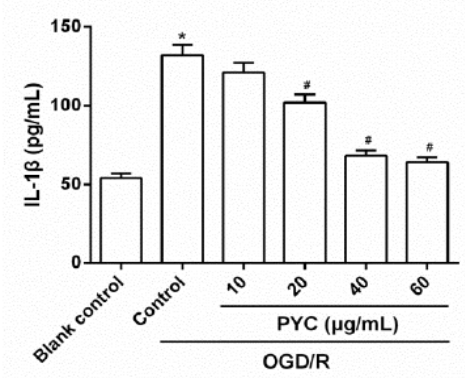

whereas PYC $(10,20,40$, and $60 \mu \mathrm{g} / \mathrm{mL})$ pretreatment resulted in a dose-dependent enhancement of SOD activity (Fig. 2A). In addition, a significant increase in ROS generation and a noticeable elevation in MDA levels (a cellular lipid peroxidation product) were found after OGD/R exposure. Conversely, PYC pretreatment decreased MDA content and ROS production in a dose-dependent manner (Fig. 2B and C). Furthermore, JC-1 was used to assess the extent of mitochondrial depolarization in astrocyte cultures. After OGD/R exposure, the red/green fluorescence intensity of JC-1 in astrocytes was reduced, suggesting that MMP dissipation was induced, whereas PYC pretreatment significantly stabilized the MMP in a dose-dependent manner (Fig. 2D). Taken together, these findings demonstrated that PYC pretreatment distinctly ameliorated OGD/R-induced oxidative stress in primary rat astrocytes. 
Fig. 4. Effects of PYC on cell apoptosis in primary rat astrocytes subjected to OGD for $6 \mathrm{~h}$ followed by reoxygenation for $24 \mathrm{~h}$. (A, B) The cell apoptosis was measured by flow cytometry. The expression levels of $(\mathrm{C}, \mathrm{E})$ Bax, Bcl-2, and (D) cleaved caspase-3 proteins were determined by western blot analysis and representative blots are shown. ${ }^{*} P<0.05$ vs. blank control group, ${ }^{\#} P<0.05$ vs. control group.

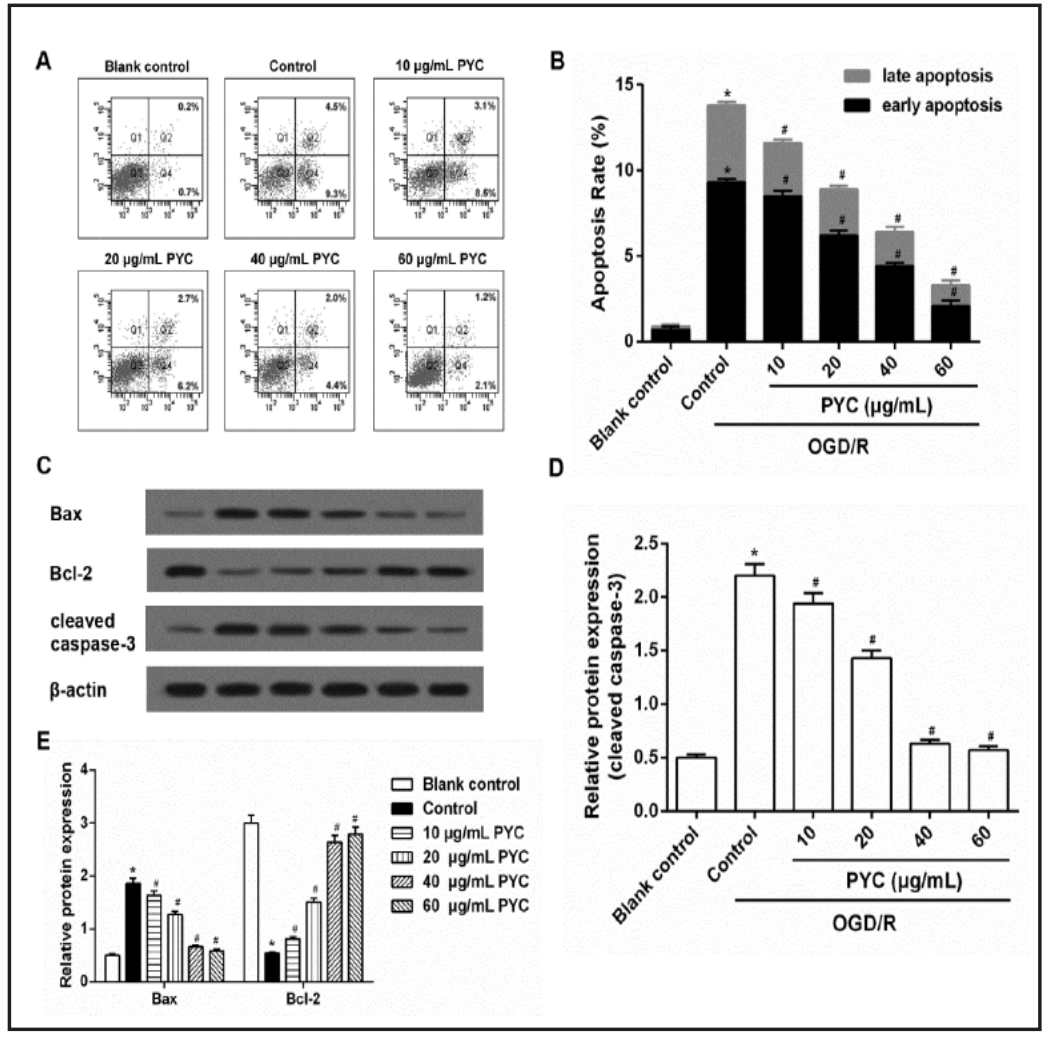

PYC pretreatment suppressed OGD/R-induced inflammatory response in primary rat astrocytes

To determine the effects of PYC on the OGD/R-induced inflammatory response in primary rat astrocytes, the concentrations of TNF- $\alpha$, IL- $1 \beta$ and IL- 6 in the culture supernatant were detected. The data revealed that the accumulation of TNF- $\alpha$, IL- $1 \beta$ and IL- 6 secretion induced by $\mathrm{OGD} / \mathrm{R}$ in conditioned medium was decreased dose-dependently following PYC $(10,20,40$, and $60 \mu \mathrm{g} / \mathrm{mL})$ pretreatment in primary rat astrocytes (Fig. 3A, B and C). These results illustrated that PYC pretreatment suppressed OGD/R-induced inflammatory response in primary rat astrocytes.

PYC pretreatment suppressed OGD/R-induced cell apoptosis in primary rat astrocytes

To assess the effects of PYC on OGD/R-induced cell apoptosis in primary rat astrocytes, the apoptosis rate and expression of apoptosis-related proteins (Bcl-2, Bax, cleaved caspase-3) were examined. The cell apoptosis rate was increased in OGD/R group, whereas the PYC treatment decreased the OGD/R induced apoptosis (Fig. 4A and 4B). Western blot analysis indicated that OGD/R exposure induced cell apoptosis with the concomitant enhancement of Bax, increase of cleaved caspase-3 expression and reduction of Bcl- 2 expression in primary rat astrocytes. These effects were reversed by PYC $(10,20,40$, and $60 \mu \mathrm{g} / \mathrm{mL})$ pretreatment in a dose-dependent manner (Fig. 4C, 4D and 4E). These data confirmed that PYC pretreatment suppressed OGD/R-induced cell apoptosis in primary rat astrocytes.

PYC pretreatment suppressed OGD/R-induced activation of NF- $K B$ and ERK1/2 pathways in primary rat astrocytes

Importantly, we evaluated the effects of PYC on NF- $\kappa B$ and ERK1/2 signaling pathways under OGD/R condition. The result showed that NF- $\mathrm{KB}$ p65 phosphorylation and the stimulation after OGD/R exposure was blunted by using PYC (Fig. 5A). Moreover, the nuclear translocation of the NF- $\kappa B$ p65 subunit was enhanced under OGD/R condition, and the enhancement was prevented largely by PYC (Fig. 5B). In addition, both the NF- $\kappa$ B activity 
Fig. 5. Effects of PYC on the NF-KB and ERK1/2 pathways in primary rat astrocytes subjected to OGD for $6 \mathrm{~h}$ followed by reoxygenation for $24 \mathrm{~h}$. (A) NF$\kappa \mathrm{B}$ activity was determined using an NF- $\kappa B$ p65 ActivELISA kit. (B) The expression levels of p-ERK1/2, ERK1/2, and $\beta$-actin proteins were determined by western blot analysis and representative blots are shown. ${ }^{*} P<0.05$ vs. blank control group, ${ }^{\#} P<0.05$ vs. control group.

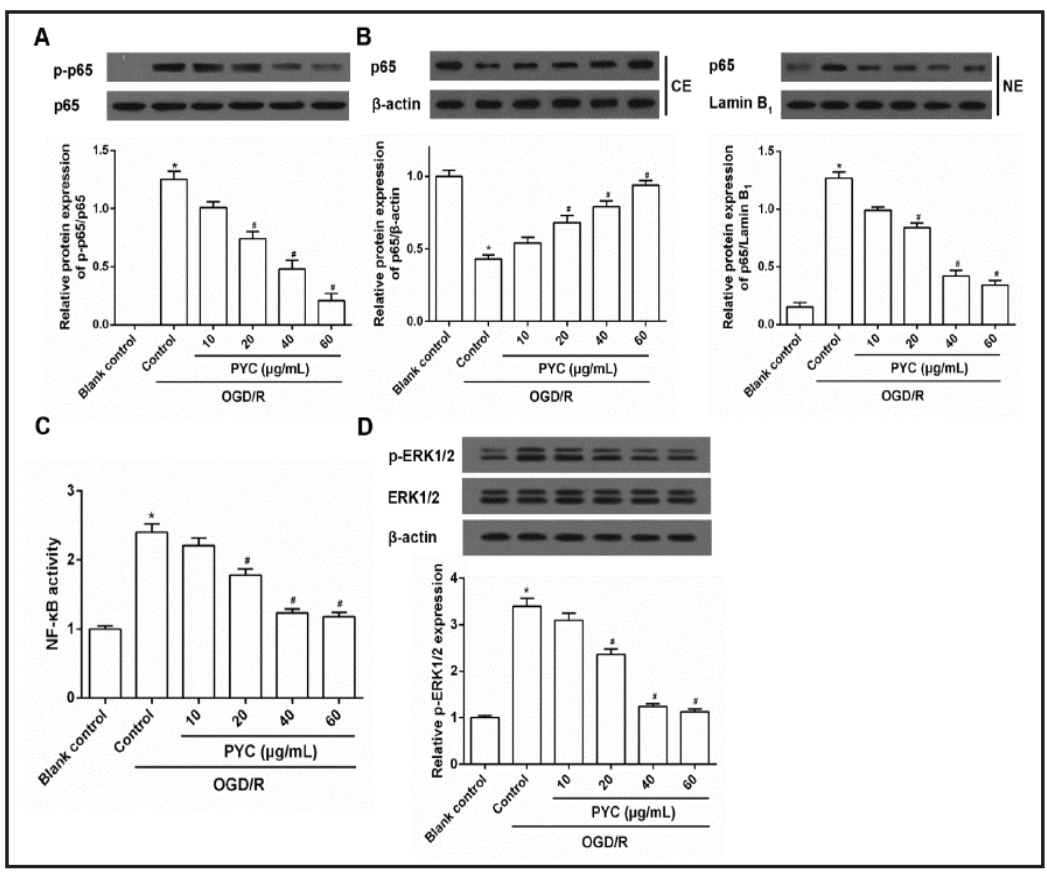

Fig. 6. Effects of PYC, NF$\kappa B$ inhibitor, and ERK1/2 inhibitor on OGD/R-induced oxidative stress and inflammation injury in primary rat astrocytes. (A) NF-KB activity was determined using an NF-KB p65 ActivELISA kit. (B) The expression levels of p-ERK1/2, ERK1/2, and $\beta$-actin proteins were determined by westerning blot analysis and representative blots are shown. The levels of (C) ROS and (D) TNF- $\alpha$ were determined using the commercial kits, respectively. ${ }^{*} P<0.05$ vs. blank control group, ${ }^{\#} P<0.05$ vs. control group.

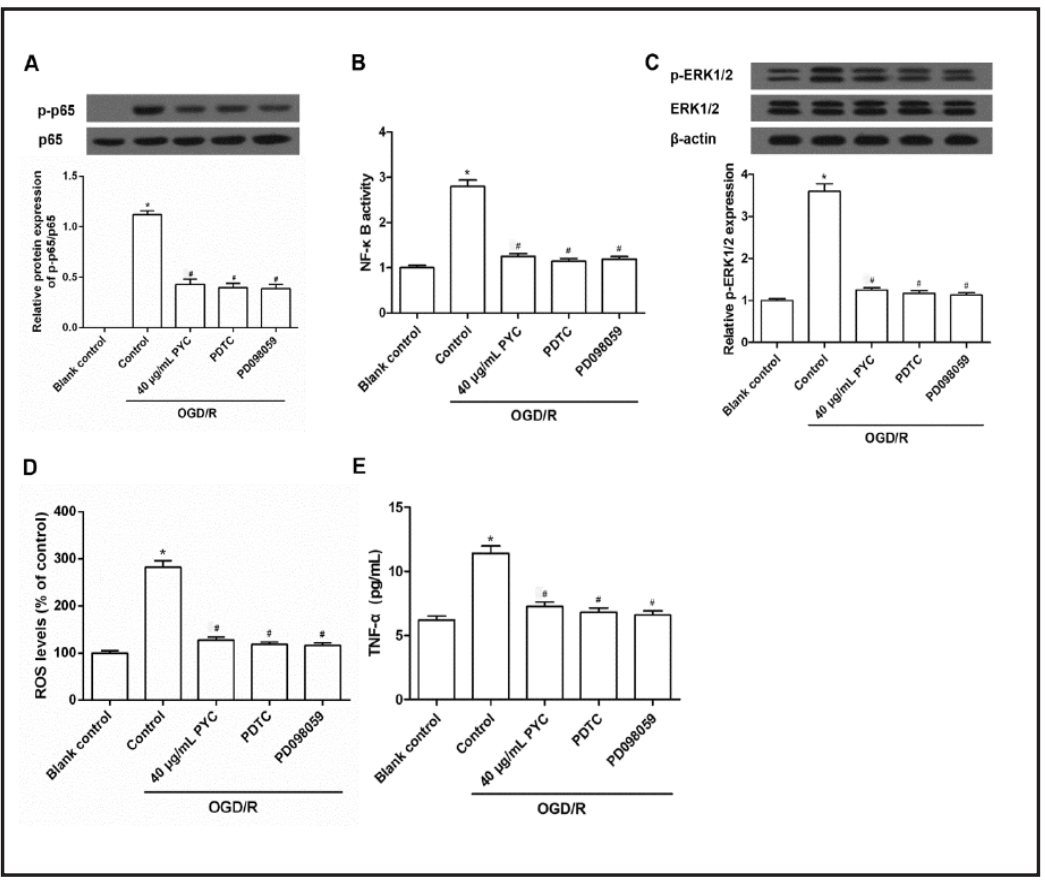

and the expression levels of p-ERK1/2 proteins were augmented after OGD/R exposure. But PYC pretreatment clearly suppressed the constitutive and inducible NF- $\mathrm{KB}$ activity and phosphorylation of ERK1/2 proteins caused by OGD/R in a dose-dependent manner (Fig. 5C and D). These findings implied that PYC pretreatment suppressed OGD/R-induced activation of NF- $\mathrm{KB}$ and $\mathrm{ERK} 1 / 2$ pathways in primary rat astrocytes.

PYC pretreatment suppressed OGD/R-induced oxidative stress and inflammation injury via NF- $\kappa B$ and ERK1/2 pathways in primary rat astrocytes

To elucidate whether NF- $\kappa \mathrm{B}$ and ERK1/2 pathways were involved in OGD/R-induced oxidative stress and inflammation injury, we used the NF- $\kappa B$ inhibitor PDTC and ERK1/2 inhibitor PD098059 to further evaluate the mechanism of action of PYC in the OGD/R- 
treated primary rat astrocytes. We observed that both PDTC and PD098059 attenuated OGD/R-induced NF- $\kappa B$ p 65 phosphorylation (Fig. 6A), NF- $\kappa$ B activity (Fig. 6B) and ERK1/2 phosphorylation (Fig. 6C)in primary rat astrocytes. Moreover, similar to the inhibitory effects of PYC, PDTC and PD098059 dramatically inhibited OGD/R-induced ROS production and TNF- $\alpha$ secretion (Fig. 6D and E). Therefore, it can be concluded that PYC pretreatment

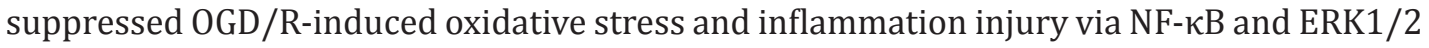
pathways in primary rat astrocytes.

\section{Discussion}

Stroke is a leading cause of death and disability, and ischemic strokes constitute most of all stroke cases and develop through interference with the blood supply to the brain. Understanding the role of astrocytes in ischemic stroke is assuming increasing prominence, not only as an important component on its own within the neurovascular unit, but also because astrocytes can influence neuronal outcome. Additionally, astrocytes have been demonstrated to protect neurons and increase their survival in many pathological settings. A large body of experimental evidence has indicated that impaired astrocytes play crucial roles in the pathological process of cerebral ischemia; therefore, astrocytes may represent a breakthrough target for neuroprotective therapeutic strategies. As a natural plant extract, PYC has been demonstrated to protective against traumatic brain injury [13] and alleviate amyloid- $\beta$ peptide-induced apoptosis of PC12 cells [24]. In addition, PYC had been reported to protect human neuroblastoma SH-SY5Y cells following acrolein-induced cytotoxicity [14]. In this study, we mainly focused on the effects of PYC on OGD/R-induced injury and the potential mechanism in primary rat astrocytes, an in vitro model of ischemic stroke.

PYC is a patented mix of bioflavonoids with strong anti-oxidative and anti-inflammatory potency that has been shown to be beneficial in multiple medical conditions. PYC appears to impede the progression of subclinical arterial lesions in low-risk asymptomatic subjects, and the reduction in plaque progression is associated with the inhibition of oxidative stress during atherosclerosis [12]. PYC supplementation plays an active role in improving the control of the most common clinical aspects of psoriasis and in decreasing oxidative stress [25]. In a rat model of traumatic brain injury, PYC protects CA3-CA1 synaptic function [26] via its free radical scavenging and antioxidant activity [27]. Notably, our results showed that PYC pretreatment suppressed OGD/R-induced oxidative stress in primary rat astrocytes, along with a reduction in MDA content and ROS production, and an increase in SOD activity and MMP.

In addition, chronic inflammation has been thought to play a major role in the onset and progression of astrocytes injury by producing pro-inflammatory mediators during ischemic stroke. PYC has been found to be effective in attenuating the release of proinflammatory cytokines, including TNF- $\alpha$, IL- 6 and IL-1 $\beta$, in lipopolysaccharide-stimulated BV2 microglia, in part via NF- $\kappa B$ inhibition [11]. PYC inhibits the inflammatory and nitrosative stress on joint inflammation in the treatment of monosodium urate crystal-induced arthritis [28]. PYC treatment can also alleviate ventilator-induced lung injury in rats through its ability to diminish the production of inflammatory cytokines, TNF- $\alpha$, IL-6 and IL-1 $\beta$ [29]. Likewise, our findings indicated that PYC pretreatment suppressed OGD/R-induced inflammatory response in primary rat astrocytes. Based on these results, we may infer that the antioxidative and anti-inflammatory properties of PYC may underlie its anti-apoptotic effects.

Previous research has shown that both a reduced rate of cell survival and an enhanced LDH release can be observed in OGD/R-treated astrocytes [30]. Astrocytes apoptosis may contribute to the pathogenesis of a number of acute and chronic neurodegenerative disorders, such as cerebral ischemia, Alzheimer's disease and Parkinson's disease [31]. Moreover, PYC treatment significantly increases cell viability and decreases cell apoptosis in rotenone-treated PC12 cells in a dose-dependent manner [32]. PYC modulates apoptosis by suppressing oxidative stress and inflammation in high glucose-treated renal tubular

\section{KARGER}


cells [10]. PYC exerts protective effects against acrolein toxicity by modulating oxidative stress in human neuroblastoma SH-SY5Y cells [14]. Similarly, our data demonstrated that PYC pretreatment attenuated OGD/R-induced loss of cell viability and the increase in cell apoptosis in primary rat astrocytes. Thus, we may conclude that the protective effects of PYC against astrocytes injury are dependent on multiple functions, including anti-oxidative stress, anti-inflammation and anti-apoptosis effects.

An improved understanding of disease mechanisms is crucial for the development of applicable treatment strategies. It has been observed that the expression of p-ERK1, p-ERK2, $\mathrm{p}-\mathrm{IKKB} \alpha / \beta$ and $\mathrm{P} 65$ proteins is upregulated in OGD/R-treated rat astrocytes [30]. Previous scholars have found that this phytochemical PYC inhibits NF- $\kappa B$ activation in preventing the atherogenic process [33]. In addition, NF- $\kappa \mathrm{B}$ activation was inhibited by PYC treatment to reduce the inflammatory response in ventilator-induced lung injury rats [29]. Our results indicate that PYC inhibit NF- $\kappa B$ activity in OGD/R-induced astrocytes in a dose-dependent manner. This results is consistent with a previously paper, which demonstrated that PYC protects PC12 cells from rotenone-induced neurotoxicity by suppressing the NF- $\mathrm{KB}$-iNOS signaling pathway [32]. Moreover, PYC was reported to suppress the activation of NF$\kappa B$ and ERK1/2 pathways in articular chondrocytes and synovial fibroblasts in vitro [28]. ROS generation can lead to ERK1/2 phosphorylation, and then activate the downstream transcriptional factor NF- $\kappa \mathrm{B}$ in brain astrocytes [34]. Previously study reported that ERK inhibitor reduced NF-KB-related inflammatory upstream pathways and decreased the levels of inflammatory end-products [35]. Thus we suspected that PYC could inhibit ERK1/2 phosphorylation. Then the suppression of ERK1/2 phosphorylation by PYC was verified in our results. Our results revealed that PYC pretreatment suppressed OGD/R-induced oxidative stress and inflammation injury via NF- $\kappa \mathrm{B}$ and ERK1/2 MAPK pathways in primary rat astrocytes.

In summary, our data suggested the effects of PYC on OGD/R-induced injury in primary rat astrocytes, which implies a potentially promising strategy for ischemic stroke treatment. Our findings revealed that PYC has neuroprotective effects against OGD/R-induced injury in primary rat astrocytes, which may be attributed to its anti-oxidant and anti-inflammatory activities via NF- $\kappa \mathrm{B}$ and ERK1/2 pathways. Overall, this fundamental research highlights the potential therapeutic application of PYC and the novel therapeutic opportunities related to inhibition of oxidative stress and inflammation in the treatment of ischemic diseases.

\section{Abbreviations}

PYC (Pycnogenol); OGD/R (oxygen-glucose deprivation/reoxygenation); SOD (superoxidedismutase); MMP(mitochondrialmembranepotential); MDA (malondialdehyde); ROS (reactive oxygen species); SD (Sprague-Dawley); ELISA (Enzyme-linked immunosorbent assay); GFAP (glial fibrillary acidic protein); LDH (lactate dehydrogenase); DCFH-DA $\left(2^{\prime}, 7^{\prime}\right.$-dichlorfluorescein-diacetate); NF- $\kappa \mathrm{B}$ (nuclear factor-kappaB); PDTC (pyrrolidine dithiocarbamate); ERK1/2 (extracellular signal-regulated kinase 1/2); FBS fetal bovine serum); PBS (phosphate buffered saline).

\section{Disclosure Statement}

The authors declare that they have no conflict of interest. 


\section{Cellular Physiology Cell Physiol Biochem 2017;42:987-998

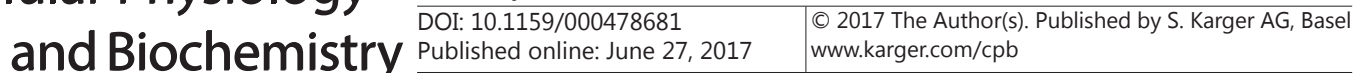

Xia et al.: Protective Effects of Pycnogenol in Rat Astrocytes

\section{References}

1 Wang C, Jie C, Dai X: Possible roles of astrocytes in estrogen neuroprotection during cerebral ischemia. Rev Neurosci 2014;25:255-268.

2 Gabryel B, Kasprowska D, Kost A, Labuzek K, Urbanek T: [Astrocytes in ischemic stroke - a potential target for neuroprotective strategies]. Postepy Hig Med Dosw (Online) 2015;69:384-397.

-3 Barreto G, White RE, Ouyang Y, Xu L, Giffard RG: Astrocytes: targets for neuroprotection in stroke. Cent Nerv Syst Agents Med Chem 2011;11:164-173.

4 Rappold PM, Tieu K: Astrocytes and therapeutics for Parkinson's disease. Neurotherapeutics 2010;7:413423.

-5 Inyushin MY, Huertas A, Kucheryavykh YV, Kucheryavykh LY, Tsydzik V, Sanabria P, Eaton MJ, Skatchkov SN, Rojas LV, Wessinger WD: L-DOPA Uptake in Astrocytic Endfeet Enwrapping Blood Vessels in Rat Brain. Parkinsons Dis 2012;2012:321406.

6 Yu S, Wang X, Lei S, Chen X, Liu Y, Zhou Y, Wu J, Zhao Y: Sulfiredoxin-1 protects primary cultured astrocytes from ischemia-induced damage. Neurochem Int 2015;82:19-27.

7 Stoll G, Kleinschnitz C, Nieswandt B: Combating innate inflammation: a new paradigm for acute treatment of stroke? Ann N Y Acad Sci 2010;1207:149-154.

8 Giffard RG, Swanson RA: Ischemia-induced programmed cell death in astrocytes. Glia 2005;50:299-306.

-9 Buz'Zard AR, Lau BH: Pycnogenol reduces talc-induced neoplastic transformation in human ovarian cell cultures. Phytother Res 2007;21:579-586.

10 Kim YJ, Kim YA, Yokozawa T: Pycnogenol modulates apoptosis by suppressing oxidative stress and inflammation in high glucose-treated renal tubular cells. Food Chem Toxicol 2011;49:2196-2201.

-11 Fan B, Dun SH, Gu JQ, Guo Y, Ikuyama S: Pycnogenol Attenuates the Release of Proinflammatory Cytokines and Expression of Perilipin 2 in Lipopolysaccharide-Stimulated Microglia in Part via Inhibition of NFkappaB and AP-1 Activation. PLoS One 2015;10:e0137837.

-12 Belcaro G, Dugall M, Hosoi M, Ippolito E, Cesarone M, Luzzi R, Cornelli U, Ledda A: Pycnogenol(R) and Centella Asiatica for asymptomatic atherosclerosis progression. Int Angiol 2014;33:20-26.

13 Scheff SW, Ansari MA, Roberts KN: Neuroprotective effect of Pycnogenol(R) following traumatic brain injury. Exp Neurol 2013;239:183-191.

14 Ansari MA, Keller JN, Scheff SW: Protective effect of Pycnogenol in human neuroblastoma SH-SY5Y cells following acrolein-induced cytotoxicity. Free Radic Biol Med 2008;45:1510-1519.

15 Bentz K, Molcanyi M, Hess S, Schneider A, Hescheler J, Neugebauer E, Schaefer U: Neural differentiation of embryonic stem cells is induced by signalling from non-neural niche cells. Cell Physiol Biochem 2006;18:275-286.

-16 Iram T, Trudler D, Kain D, Kanner S, Galron R, Vassar R, Barzilai A, Blinder P, Fishelson Z, Frenkel D: Astrocytes from old Alzheimer's disease mice are impaired in Abeta uptake and in neuroprotection. Neurobiol Dis 2016;96:84-94.

17 Gwag BJ, Lobner D, Koh JY, Wie MB, Choi DW: Blockade of glutamate receptors unmasks neuronal apoptosis after oxygen-glucose deprivation in vitro. Neuroscience 1995;68:615-619.

- 18 Ohkawa H, Ohishi N, Yagi K: Assay for lipid peroxides in animal tissues by thiobarbituric acid reaction. Anal Biochem 1979;95:351-358.

19 Wu W, Xia Q, Luo RJ, Lin ZQ, Xue P: In vitro Study of the Antagonistic Effect of Low-dose Liquiritigenin on Gemcitabine-induced Capillary Leak Syndrome in Pancreatic Adenocarcinoma via Inhibiting ROSMediated Signalling Pathways. Asian Pac J Cancer Prev 2015;16:4369-4376.

20 Reers M, Smith TW, Chen LB: J-aggregate formation of a carbocyanine as a quantitative fluorescent indicator of membrane potential. Biochemistry 1991;30:4480-4486.

21 Zhu XJ, Shi Y, Peng J, Guo CS, Shan NN, Qin P, Ji XB, Hou M: The effects of BAFF and BAFF-R-Fc fusion protein in immune thrombocytopenia. Blood 2009;114:5362-5367.

22 Liu L, You Q, Tu Y, Li Q Zheng L, Li X, Gu J, Wang G: Midazolam inhibits the apoptosis of astrocytes induced by oxygen glucose deprivation via targeting JAK2-STAT3 signaling pathway. Cell Physiol Biochem 2015;35:126-136.

23 Guo R, Wu K, Chen J, Mo L, Hua X, Zheng D, Chen P, Chen G, Xu W, Feng J: Exogenous hydrogen sulfide protects against doxorubicin-induced inflammation and cytotoxicity by inhibiting p38MAPK/NFkappaB pathway in H9c2 cardiac cells. Cell Physiol Biochem 2013;32:1668-1680. 


\section{Cellular Physiology Cell Physiol Biochem 2017;42:987-998 and Biochemistry Published online: June 27, $2017 \quad \begin{aligned} & \text { DOI 10159/2017 The Author(s). Published by S. Karger AG, Basel } \\ & \text { www.karger.com/cpb }\end{aligned}$ \\ Xia et al.: Protective Effects of Pycnogenol in Rat Astrocytes}

24 Peng QL, Buz'Zard AR, Lau BH: Pycnogenol protects neurons from amyloid-beta peptide-induced apoptosis. Brain Res Mol Brain Res 2002;104:55-65.

-25 Belcaro G, Luzzi R, Hu S, Cesarone MR, Dugall M, Ippolito E, Corsi M, Caporale S: Improvement in signs and symptoms in psoriasis patients with Pycnogenol(R) supplementation. Panminerva Med 2014;56:41-48.

26 Norris CM, Sompol P, Roberts KN, Ansari M, Scheff SW: Pycnogenol protects CA3-CA1 synaptic function in a rat model of traumatic brain injury. Exp Neurol 2016;276:5-12.

27 Ozer Sehirli A, Sener G, Ercan F: Protective effects of pycnogenol against ischemia reperfusion-induced oxidative renal injury in rats. Ren Fail 2009;31:690-697.

-28 Peng YJ, Lee CH, Wang CC, Salter DM, Lee HS: Pycnogenol attenuates the inflammatory and nitrosative stress on joint inflammation induced by urate crystals. Free Radic Biol Med 2012;52:765-774.

29 Xia YF, Zhang JH, Xu ZF, Deng XM: Pycnogenol, a compound isolated from the bark of pinus maritime mill, attenuates ventilator-induced lung injury through inhibiting NF-kappaB-mediated inflammatory response. Int J Clin Exp Med 2015;8:1824-1833.

30 Wang L, Zhang Y, Asakawa T, Li W, Han S, Li Q, Xiao B, Namba H, Lu C, Dong Q: Neuroprotective effect of neuroserpin in oxygen-glucose deprivation- and reoxygenation-treated rat astrocytes in vitro. PLoS One 2015;10:e0123932.

31 Takuma K, Baba A, Matsuda T: Astrocyte apoptosis: implications for neuroprotection. Prog Neurobiol 2004;72:111-127.

-32 Gao B, Chang C, Zhou J, Zhao T, Wang C, Li C, Gao G: Pycnogenol Protects Against Rotenone-Induced Neurotoxicity in PC12 Cells Through Regulating NF-kappaB-iNOS Signaling Pathway. DNA Cell Biol 2015;34:643-649.

33 Peng Q, Wei Z, Lau BH: Pycnogenol inhibits tumor necrosis factor-alpha-induced nuclear factor kappa B activation and adhesion molecule expression in human vascular endothelial cells. Cell Mol Life Sci 2000;57:834-841.

-34 Yang CM, Lin CC, Hsieh HL: High-Glucose-Derived Oxidative Stress-Dependent Heme Oxygenase-1 Expression from Astrocytes Contributes to the Neuronal Apoptosis. Mol Neurobiol 2017;54:470-483.

35 Yang XL, Kim CK, Kim TJ, Sun J, Rim D, Kim YJ, Ko SB, Jang H, Yoon BW: Anti-inflammatory effects of fimasartan via Akt, ERK, and NFkappaB pathways on astrocytes stimulated by hemolysate. Inflamm Res 2016;65:115-123. 